\title{
Optimising cognitive load and usability to improve the impact of e-learning in medical education
}

\author{
M R Davids, ${ }^{1} \mathrm{MB}$ ChB, FCP (SA), MMed (Int Med), PhD; M L Halperin, ${ }^{2}$ BSc (Biochemistry), MDCM, FRCP(C), MD; \\ U M E Chikte, ${ }^{3}$ BChD, DHSM, MDent, MSc, $\mathrm{PhD}$ \\ ${ }^{1}$ Division of Nephrology, Department of Medicine, Faculty of Medicine and Health Sciences, Stellenbosch University, Cape Town, South Africa \\ ${ }^{2}$ Emeritus Professor, Division of Nephrology, Li Ka Shing Knowledge Institute, St Michael's Hospital and University of Toronto, Canada \\ ${ }^{3}$ Department of Interdisciplinary Health Sciences, Faculty of Medicine and Health Sciences, Stellenbosch University, Cape Town, South Africa
}

Corresponding author: M R Davids (mrd@sun.ac.za)

E-learning has the potential to support the development of expertise in clinical reasoning by being able to provide students with interactive learning experiences, exposure to multiple cases, and opportunities for deliberate practice with tailored feedback. This review focuses on two important but underappreciated factors necessary for successful e-learning, i.e. the management of the learner's cognitive load and the usability of the technology interface.

Cognitive load theory views learning as involving active processing of information by working memory via separate visual and auditory channels. This system is of very limited capacity and any cognitive load that does not directly contribute to learning is considered extraneous and likely to impede learning. Researchers in cognitive load theory have provided evidence-based instructional design principles to reduce extraneous cognitive load and better manage the cognitive processing necessary for learning.

Usability is a concept from the field of human-computer interaction which describes how easy technology interfaces are to use, and is routinely evaluated and optimised in the software development industry. This is seldom the case when e-learning resources are developed, especially in the area of medical education. Poor usability limits the potential benefit of educational resources, as learners experience difficulties with the technology interface while simultaneously dealing with the challenges of the content presented. Practitioners in the field of human-computer interaction have provided guidelines and methods for evaluating and optimising the usability of e-learning materials.

The fields of cognitive load theory and human-computer interaction share a common goal in striving to reduce extraneous cognitive load. The load induced by poor usability of e-learning materials can be viewed as a specific component of extraneous cognitive load, adding to any load resulting from poor instructional design. The guidelines from these two fields are complementary and, if correctly implemented, may substantially improve the impact of our e-learning resources on the development of the clinical reasoning skills of students.

Afr J Health Professions Educ 2015;7(2):147-152. DOI:10.7196/AJHPE. 569

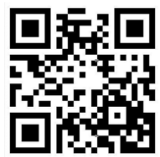

Clinical reasoning ability is central to clinical competence as it allows medical practitioners to evaluate patients and their test results in order to make accurate diagnoses and implement appropriate treatment. ${ }^{[1]}$ The development of expertise in clinical reasoning may be facilitated through active learning and practice with authentic cases. E-learning has the potential to support this process by providing students with interactive learning experiences, exposure to multiple cases, and opportunities for deliberate practice with tailored feedback.

Creative educators use animation and simulation to build innovative learning resources. The available technology makes it possible to offer personalised instruction, collaboration and an engaging, even immersive, learning experience. ${ }^{[2]}$ Simulations allow inexperienced trainees to practise their clinical reasoning skills on virtual patients without exposing real patients to the possibility of harm, and can provide exposure to a variety of clinical presentations and uncommon medical conditions. Errors in managing these virtual patients may be allowed and provide valuable learning opportunities.

Developing innovative e-learning materials can, however, be very time consuming and expensive. For example, a survey published in 2007 on the development of computer-based virtual patients at medical schools in the USA and Canada revealed that each took an average of 16.6 months to complete and 85\% cost >USD10 000 (ZAR120 100). ${ }^{[3]}$ This level of investment of time and money needs to be justified by the educational impact of the resources developed, especially in under-resourced environments such as African tertiary education institutions.

There are many factors that must be in place to ensure successful e-learning. ${ }^{[4]}$ This article highlights two critically important but under-appreciated factors, i.e. the management of the learner's cognitive load and the usability of the computer interface. The sections that follow briefly discuss: (i) development of expertise in clinical reasoning; (ii) cognitive load theory and its relevance to e-learning; and (iii) importance of the usability of computer interfaces.

\section{Developing expertise in clinical reasoning}

Learning requires alterations in long-term memory. The major mechanisms involved are the acquisition and automation of knowledge schemas. ${ }^{[5]}$ In the context of clinical medicine, schemas are sometimes also referred to as 'illness scripts. ${ }^{[6]}$ These are cognitive constructs or 'mental models' for organising and storing information.

The critical role of long-term memory in intellectual performance started emerging with the publication in the 1960 s of studies on chess players. ${ }^{[7]}$ After a brief exposure to a typical mid-game position, expert chess players were much better than novices in their ability to recall the exact positions of the pieces. However, when the same pieces were randomly distributed on the board, there was no difference between experts and novices in recalling the positions of the pieces. ${ }^{[8]}$ Chess experts do not see a position as isolated pieces, but as configurations of pieces and squares, most of which they have seen many times before. These configurations are stored as 'chunks' or 
schemas, and it is estimated that chess experts have between 10000 and 100000 such configurations stored in their long-term memory.

Similarly, medical experts appear to solve most clinical problems by pattern recognition, without resorting to analytical, pathophysiological reasoning. ${ }^{[9]}$ They are able to do this, with good diagnostic accuracy, by drawing on an extensive database of schemas or illness scripts stored in their long-term memory. Many studies on noviceexpert differences indicate that expertise is the result of the acquisition of a large fund of domainspecific knowledge that is well organised and easily retrieved when needed ${ }^{[10]}$ Deliberate practice over an extended period of time produces a high level of automation of these schemas ${ }^{[1]}$ so that their use no longer requires conscious processing and makes minimal demands on the limited resources of working memory. This is how the expert clinician is able to make diagnoses rapidly, accurately and effortlessly. However, when problems are unusual or complex, the expert is able to shift from pattern recognition to analytical reasoning and bring to bear an extensive store of basic science knowledge to address the problem. ${ }^{[12]}$ This is often required in disciplines such as anaesthesiology, intensive care medicine and nephrology, which are rooted in the basic sciences. $^{[12]}$

Expertise in clinical reasoning is very case specific. ${ }^{[13]}$ Our challenge as teachers is to help students develop expertise that can be effectively applied to the diagnosis and management of different - but related - cases that they encounter later. Such transfer of expertise is very difficult to achieve, ${ }^{[13,14]}$ but may be facilitated by ensuring active learning and creating opportunities for 'deliberate practice'[11] with carefully selected and sequenced examples. Multiple examples of cases or clinical problems allow learners to encounter key concepts in a variety of contexts. This facilitates the abstraction of the underlying concepts rather than merely focusing on the surface features of clinical problems, and improves the transfer of clinical reasoning ability from one problem to another. ${ }^{[1]}$

E-learning offers the possibility of fostering deep learning and the transfer of expertise in clinical reasoning by being able to provide students with interactive learning experiences, exposure to multiple cases, including cases seldom encountered during their clinical rotations, and opportunities for deliberate practice with immediate feedback.

There are many factors that must be in place to ensure successful e-learning ${ }^{[4]}$ and exploit the educational potential of innovative e-learning resources. These include ensuring institutional buy-in, ensuring that appropriate hardware and software are available, and providing skills training and technical and administrative support. There must be appropriate integration of e-learning into the curriculum, ensuring that assessments include the e-learning material, and a blended learning approach should be used where possible.

The following sections highlight the importance of managing the learner's cognitive load and optimising the usability of the computer interface when implementing e-learning. These factors may have a major influence on the benefit derived from the e-learning resources we employ to develop the clinical reasoning skills of our students.

\section{Managing cognitive load to promote learning}

Cognitive load theory builds on well-established models of human memory that include the subsystems of sensory memory, working memory and long-term memory. ${ }^{[15]}$ While long-term memory appears to have an unlimited capacity, working memory has a very limited capacity and can hold and process only a few discrete elements at any given time. ${ }^{[16]}$

Sweller's cognitive load theory ${ }^{[17]}$ and Mayer's cognitive theory of multimedia learning ${ }^{[18]}$ are based on a model of human cognitive architecture that views learning as involving the active processing of information by working memory via separate visual and auditory channels (Fig. 1). This system for dealing with new information is of very limited capacity and any cognitive load that does not directly contribute to learning is considered extraneous and likely to impede learning.

Overloading the limited capacity of working memory is more likely to occur when the content to be learned is difficult and presents a high intrinsic cognitive load. ${ }^{[19]}$ Intrinsic cognitive load refers to the essential processing required to understand the learning material. When the material consists of multiple interacting elements of information, the intrinsic cognitive load will be high and learners therefore experience it as difficult. Because of the interaction the elements cannot be learned in isolation or sequentially, but must be assimilated simultaneously for learning to occur. ${ }^{[19]}$ The topic of metabolic acidosis, for example, is difficult because there are many interacting elements that operate simultaneously. Students have to appreciate that metabolic acidosis may be caused by a gain of acid or the loss of sodium bicarbonate. They must also appreciate the role of the following: the extracellular fluid volume in determining the bicarbonate concentration; buffer systems in ameliorating the effects of an acid load; the kidney in excreting acid and generating new bicarbonate; and the respiratory system, which compensates for the acidosis by increasing ventilation.

In contrast to the example of metabolic acidosis, some content may also contain many elements, but because of a low level of interactivity these elements can be learned in isolation or sequentially. The intrinsic cognitive load which it presents is low, and the content is therefore not experienced as difficult. For example, learning where hormones are produced could involve a long list of hormones and their sites of origin, but each of these unrelated hormone-origin pairs has little interaction with the successful learning of any other pair. Learning that insulin is produced by the pancreas, for instance, can be done in isolation and without reference to any other hormone and its site of origin.

The number of elements and degree of interactivity in a particular piece of learning material can only be an estimate as it varies according to the level of expertise of an individual.

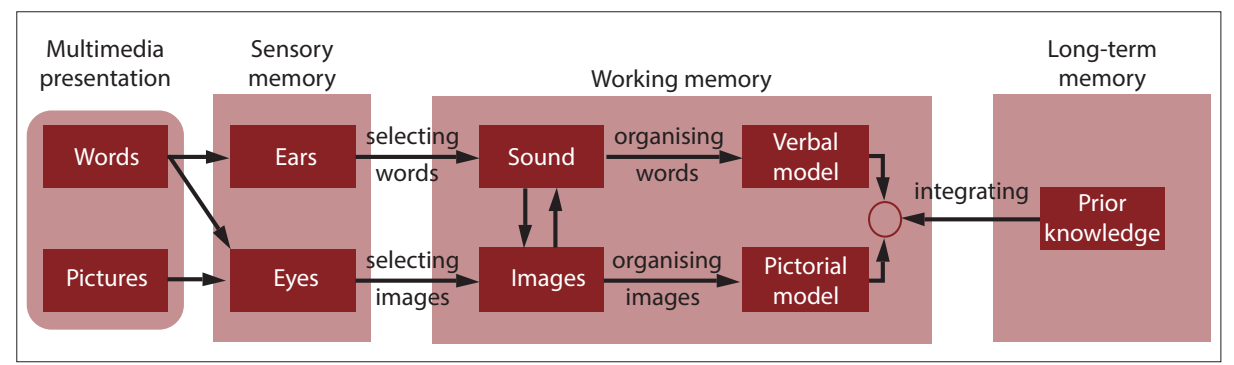

Fig. 1. The cognitive theory of multimedia learning. Sensory memory holds an exact copy of what was presented for $<0.25$ second, working memory holds a more processed version for $<30$ seconds and can process only a few items at any one time, and long-term memory has virtually unlimited capacity, holding an individual's entire store of knowledge for long periods of time. From Mayer RE, ${ }^{[20]}$ with permission. 
Therefore, whether material is experienced as difficult or not depends mainly on the presence, sophistication and automation of pre-existing schemas in the long-term memory of the individual.

There is a growing body of evidence supporting the idea that learning materials should be designed consistent with principles of cognitive load theory. Research-based design principles have been proposed which are aimed at reducing extraneous cognitive load, managing essential processing, and fostering generative processing during learning. These principles are listed in Table 1 and discussed in the sections below.

\section{Reducing extraneous processing}

Extraneous cognitive load is caused by poor instructional design and results in processing being required that does not contribute to schema acquisition or automation. Methods of presentation that reduce extraneous cognitive load free up working memory and facilitate learning. Mayer ${ }^{[20]}$ has recommended reducing extraneous load by applying the coherence principle, which states that all irrelevant material should be eliminated; the signalling principle, which involves highlighting essential material; and the spatial contiguity principle, which involves placing printed words near the corresponding graphics. These principles are aimed at minimising the splitting of the learners' attention between multiple sources of information, and avoiding the presentation of redundant or irrelevant information. ${ }^{[5]}$ For example, if a set of images illustrating the functions of the kidneys is physically separated from the corresponding explanatory text, the learner needs to scan back and forth to mentally integrate these two sources of information. Restructuring the information so that the explanatory text is close to, or even inserted into, each image eliminates the need for mental integration and reduces cognitive load.

\section{Managing essential processing}

Essential processing involves selecting relevant information and representing it in working memory. The complexity of the material and the existing expertise of the learner determine the intrinsic cognitive load related to this part of the learning process. Intrinsic cognitive load can be managed by applying research-based design principles. The pretraining principle states that people learn better from a multimedia lesson when they are already familiar with the

Table 1. Principles for the design of e-learning materials from Mayer's cognitive theory of multimedia learning ${ }^{[20]}$

\begin{tabular}{|c|c|}
\hline $\begin{array}{l}\text { Reducing extraneous } \\
\text { processing }\end{array}$ & $\begin{array}{l}\text { Coherence principle: eliminate all extraneous material } \\
\text { Signalling principle: highlight essential material } \\
\text { Contiguity principle: place printed words near corresponding graphics }\end{array}$ \\
\hline $\begin{array}{l}\text { Managing essential } \\
\text { processing }\end{array}$ & $\begin{array}{l}\text { Pretraining principle: provide pretraining in the names and characteristics of } \\
\text { key concepts } \\
\text { Segmenting principle: break lessons into learner-controlled segments } \\
\text { Modality principle: present words in spoken form rather than as text to be read }\end{array}$ \\
\hline $\begin{array}{l}\text { Fostering generative } \\
\text { processing }\end{array}$ & $\begin{array}{l}\text { Multimedia principle: use words and pictures rather than words alone } \\
\text { Personalisation principle: present words in a conversational style } \\
\text { Voice principle: use a human voice rather than a machine voice }\end{array}$ \\
\hline
\end{tabular}

key components and concepts. Novice learners should therefore receive pretraining in the names and functions of each major component of a new lesson. The segmenting principle states that people learn better when a large lesson is broken down into smaller, learner-paced segments. The navigation of the lesson should therefore be under the control of the learner. The modality principle states that the words in a multimedia lesson should be delivered via narration rather than being printed, thereby shifting information from a potentially overloaded visual channel onto the auditory channel.

\section{Fostering generative processing}

Generative processing is aimed at making sense of the material during learning and involves organising and integrating pictures and words and information from long-term memory. Three design principles are helpful for increasing motivation and engagement, thereby promoting generative processing. The multimedia principle is that people learn better from words and pictures than from words alone. For example, instead of only presenting a block of text explaining how the kidney works, add a series of illustrations. The personalisation principle is that people are more engaged and learn better when words are delivered in conversational language rather than in more formal language. For example, it is better to refer to 'your kidney' rather than 'the kidney'. The voice principle is that people learn better from multimedia lessons when the narration uses a human voice rather than a computer-generated one.

The advances in cognitive science summarised above provide useful guidance for designing effective e-learning resources that can support the development of students' clinical reasoning skills. The implementation of these design principles has been tested in multiple experiments and shown to have a significant positive impact on learning, with medium to large effect sizes. ${ }^{[20]}$

\section{Improving the usability of computer interfaces}

Usability is a concept from the discipline of human-computer interaction that describes how easy it is to use technology interfaces. ${ }^{[21]}$ Interfaces should be designed to be intuitive and self-evident, so that even inexperienced users can accomplish tasks successfully. The International Standard, ISO 9241-11, formally defines usability as the 'extent to which a product can be used by specified users to achieve specified goals with effectiveness, efficiency and satisfaction in a specified context of use. ${ }^{[22]}$

Design approaches that evaluate and optimise usability are common in the field of software development, but this is still seldom the case with e-learning, especially in the area of medical education. ${ }^{[23]}$ Studies on e-learning interventions in the health sciences rarely describe usability evaluation that has been conducted at an early stage of the development process, and usability is usually not even mentioned as a component of the final evaluation. It has been suggested that journals should encourage the authors of e-learning articles to report on usability and share the important lessons learnt, thereby helping colleagues to avoid costly mistakes in the future. ${ }^{[23]}$

High usability of e-learning materials is required to ensure the maximum educational impact, especially when the material to be learnt is complex. ${ }^{[19]}$ Poor usability limits the potential benefit obtained from e-learning resources ${ }^{[2,24]}$ by imposing an extraneous cognitive load, as users struggle with the interface and challenges of the content presented. We observed this with an interactive simulation we developed for improving clinical reasoning in the area of electrolyte 
disorders. ${ }^{[25]}$ Formal usability evaluation revealed that serious usability problems rendered the resource unusable for many participants. ${ }^{[26]}$

Some researchers have found significant learning effects from optimising the usability of learning materials. ${ }^{[27]}$ This is most likely to be seen with novice learners who experience the content as presenting a high intrinsic cognitive load and would therefore be more sensitive to any extraneous load imposed by poor usability. ${ }^{[17]}$ Other researchers have reported improvements in efficiency, satisfaction or motivation. ${ }^{[24,28,29]}$ These effects are important in the light of the high dropout rate from e-learning courses. ${ }^{[30]}$ Motivated and self-regulated learners are more likely to persist and succeed in e-learning environments, and by optimising usability one can make an important contribution to their satisfaction and motivation. An example of a usability problem and how it might be addressed is presented in Fig. 2 .

The two main approaches to usability evaluation are 'usability inspection' and 'user testing. ${ }^{[33,34]}$ Usability inspection involves a process where experts evaluate the application against established design principles and includes methods such as heuristic evaluation, cognitive walkthroughs, guideline review and consistency inspection. ${ }^{[33]}$ Cognitive walkthroughs involve evaluators doing a step-by-step execution of common tasks, taking into account a typical user's likely goals and knowledge. They focus on the differences between the user's expectations and likely actions, and the steps required by the interface. Guideline reviews check whether an interface conforms to a set of design guidelines, such as an industry standard or corporate style guide. Consistency inspection is a methodical review for consistency in design throughout an application, including the graphics, text and the interaction or navigation style.

Heuristic evaluation is the most widely used inspection method and involves experts evaluating a technology interface against a set of heuristics, or principles of good interface $\operatorname{design}^{[35]}$ (Table 2). It provides an efficient alternative to testing with representative endusers $^{[31]}$ and can usually be conducted in less time, and at much less expense, than user testing. A group of inspectors is required, as the average problem detection rate of individual inspectors is generally low and each tends to uncover a different set of usability problems. ${ }^{[36]}$ Inspectors will usually categorise the problems detected with regard to their severity and may also suggest solutions to the problems identified.
Empirical user testing involves the recruitment of typical end-users and studying their interaction with the application. This approach is often considered to have better validity and a greater impact on product development than inspection methods. ${ }^{[34,38]}$ Evaluations may be conducted in sophisticated usability laboratories and informal settings using paper prototypes and think-aloud protocols. Selecting which usability measures to use can be difficult. There is no single global measure of usability. Some measures are subjective and others objective; all have their own cost and time requirements, and examine a particular aspect of usability. The objective measures include parameters such as successful task completion and error rates, while subjective measures include parameters such as satisfaction and perceived workload ${ }^{[39]}$ and often make use of standardised questionnaires.

Recently, there has been an increasing trend to use a broader range of measures to evaluate the user experience. This includes measures such as engagement, motivation, aesthetics, and fun. ${ }^{[2,30]}$ The affective features of instructional messages can influence the level of learner motivation and engagement in deep processing. We should therefore consider incorporating instructional design features aimed at priming motivation, while being careful not to overload the learner's working memory. ${ }^{[40]}$

Our own experience ${ }^{[25,26,31,32]}$ has illustrated how clinician-teachers who are not usability

Table 2. Principles of good interface design. These commonly used heuristics are from Nielsen, ${ }^{[35]}$ with the last item from Karat et al. ${ }^{[37]}$

\begin{tabular}{ll}
\hline Heuristic & Descriptor \\
\hline 1. Visibility of system status; feedback & $\begin{array}{l}\text { Keep users informed through timely appropriate } \\
\text { feedback. They should always know where they are, } \\
\\
\end{array}$ \\
& $\begin{array}{l}\text { which actions can be taken and how these actions can be } \\
\text { performed. }\end{array}$
\end{tabular}

2. Match with the real world language, conventions

3. Consistency and conformity to standards

4. Minimise memory load; recognition rather than recall

5. Aesthetic and minimalistic design

6. Help and documentation

7. User control and freedom

8. Flexibility and efficiency of use

9. Error prevention and tolerance

10. Help users recognise, diagnose and recover from errors

11. Intuitive visual layout
Speak the users' language, with familiar words, phrases and concepts. Follow real-world conventions, making information appear in a natural and logical order.

Words, situations and actions mean the same thing; application uses commonly accepted platform conventions and conforms to user expectations.

Make objects, actions, and options visible. The user should not have to remember information from one part of the application to another. Instructions should be visible or easily retrievable.

No irrelevant information as it competes with relevant information and diminishes its relative visibility. Animation and transitions should be used sparingly.

It is better if the system can be used without documentation. If required it should be concise, easy to search and task centred.

The user can control the direction and pace of the application. Clearly marked exits are available if they take incorrect options by mistake. The application supports Undo and Redo.

Users can modify the application to suit their individual capabilities and needs, e.g. by using shortcuts.

Careful design to prevent errors occurring. Despite user errors, the intended result may still be achieved by error correction or good error management.

Error messages should be expressed in plain language (no codes), precisely indicate the problem, and constructively suggest a solution.

Position elements on screen to be easily perceived and understood, and visually attractive. 


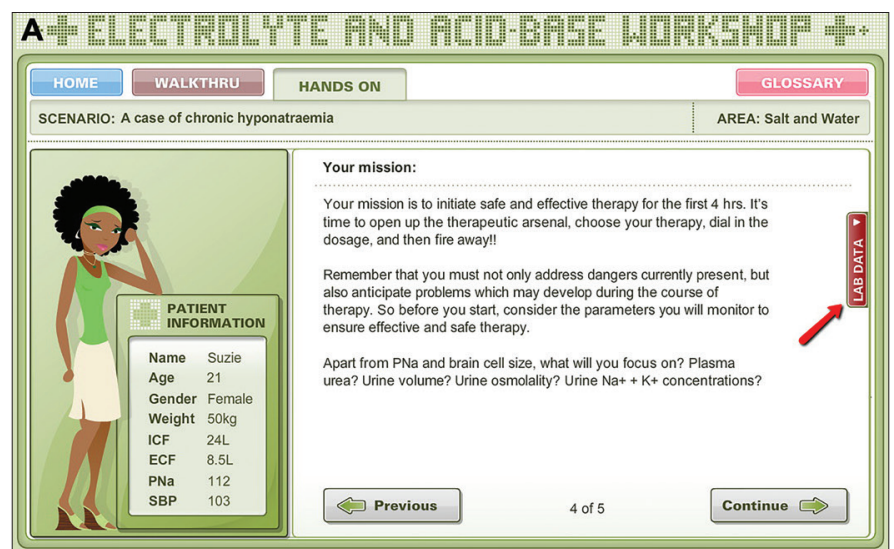

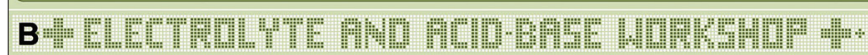

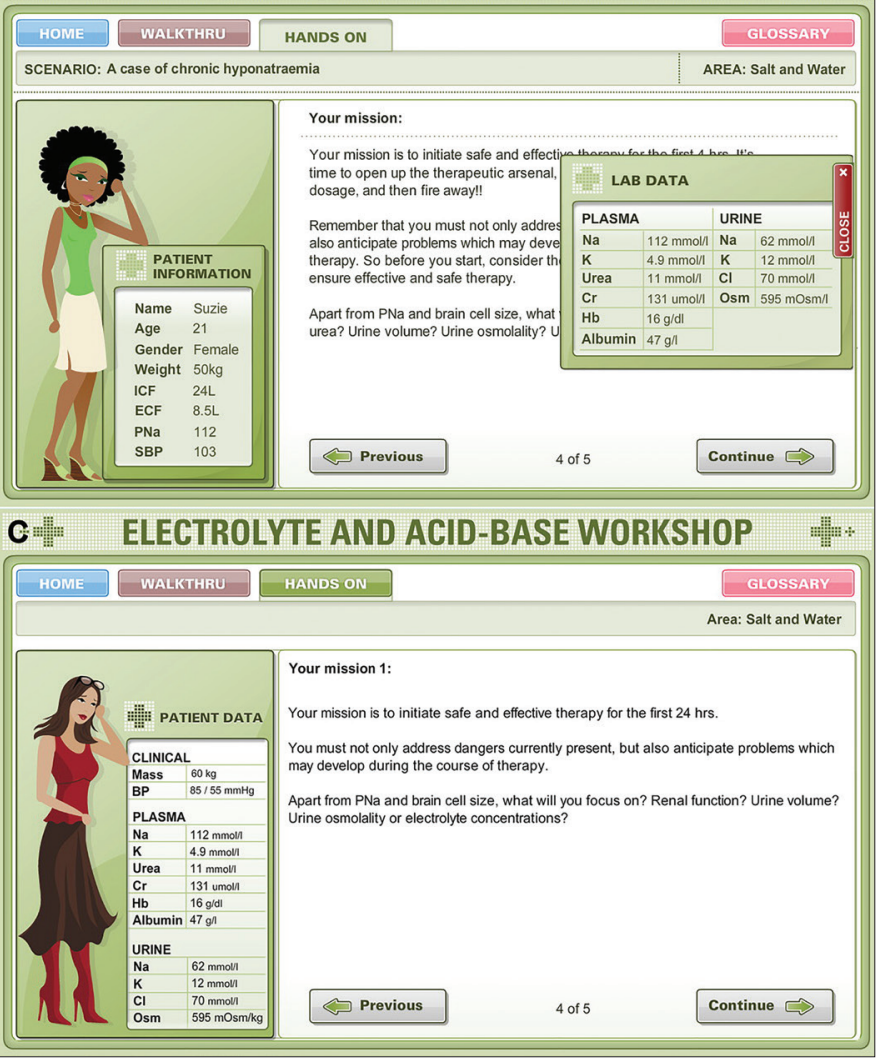

Fig. 2. An example of a usability problem. The 'hidden' laboratory data panel displays information essential for the assessment of the case presented. Upon clicking the tab at the side of the screen (A, red arrow) it slides open (B). This was completely missed by several users who then entered the treatment simulation which followed and attempted to treat their patient without having accessed this important information. When open, the panel obscures other on-screen information and remains open even when navigating to another slide. The tab has to be clicked again to close the panel. This usability problem was corrected in a subsequent redesign (C). The sliding panel has been completely eliminated and all the information is now in full view in the Patient Data panel on the left of the slide. From Davids MR, et al. ${ }^{[26,31,32]}$

experts can set about improving the usability of the resources they develop and provide a practical example for teachers in medical education and other areas. We developed a multimedia e-learning resource for electrolyte and acid-base disorders, ${ }^{[25]}$ and then conducted a usability evaluation that included testing with end-users ${ }^{[26]}$ and inspection by experts against principles of good design. ${ }^{[31]}$ Serious usability problems were identified, which limited the educational impact of the resource. User testing and expert evaluation each detected problems that were missed with the other method. We also observed a striking disconnect between objective usability measures and self-reported data. The usability problems were corrected in a subsequent redesign and resulted in substantial improvements in usability as assessed in a randomised trial that compared the original with the revised version. ${ }^{[32]}$

The question of how many users are required for an evaluation is important because each additional user adds to the cost and the time required. Nielsen ${ }^{[41]}$ has suggested that 4 - 5 users are sufficient, as they will uncover $80 \%$ of the usability problems with a technology interface. This well-known 'five users are enough' approach assumes that a formative evaluation is being conducted where several iterations of testing and redesign are envisaged. However, when the application is complex or when testing is done after the most obvious problems are already fixed, the probability of detecting each usability problem decreases and more users may be required. ${ }^{[26,42]}$

To date, there has been surprisingly limited interaction between usability practitioners and researchers in the field of cognitive load theory. A recent review $^{[43]}$ reported that cognitive load theory concepts were mentioned in very few of the citations in the Guide to Computing Literature ${ }^{[44]}$ database. The authors of this review point out that the two fields have much in common, notably a strong focus on the reduction of extraneous cognitive load. They propose that the load induced by poor usability of e-learning interfaces be viewed as a specific component of extraneous cognitive load, adding to the load resulting from poor instructional design.

\section{Conclusions and recommendations for e-learning design}

The guidelines that the fields of cognitive load theory and humancomputer interaction have provided are complementary. Both fields have a strong focus on reducing extraneous cognitive load. Applying evidencebased design principles to manage cognitive load and optimising usability is essential to improve the educational impact of our e-learning resources. This is especially relevant with innovative and interactive multimedia resources, which are very costly and time consuming to develop but have great potential in facilitating the development of the clinical reasoning skills of our students.

Usability evaluation is critical and should form a routine part of the development and implementation of e-learning materials, modules or programmes. Failing to do this may result in the implementation of resources that are unusable for many learners. It is advisable to start with the earliest versions of the resource, ideally at the prototype stage, when making changes is easier and much less costly. An iterative approach should be followed, with several cycles of testing and redesign. Heuristic evaluation by experts should be used first and, once the obvious problems have been identified and fixed, followed by testing with real users. User testing should always include the study of objective usability measures and not rely only on self-reported measures of user satisfaction.

\section{References}

1. Norman G. Research in clinical reasoning: Past history and current trends. Med Educ 2005;39:418-427 2. Ellaway R, Masters K. AMEE Guide 32: e-Learning in medical education Part 1: Learning, teaching and assessment. Med Teach 2008;30:455-473. [http://dx.doi.org/10.1080/01421590802108331]

3. Huang G, Reynolds R, Candler C. Virtual patient simulation at US and Canadian medical schools. Acad Med 2007;82:446-451. 
4. Childs S, Blenkinsopp E, Hall A, Walton G. Effective e-learning for health professionals and students - barriers and their solutions. A systematic review of the literature - findings from the HeXL project. Health Info Libr ] 2005:22:20-32.

5. Sweller J. Implications of cognitive load theory for multimedia learning. In: Mayer RE, ed. The Cambridge Handbook of Multimedia Learning. New York, NY: Cambridge University Press, 2005:19-30.

6. Barrows HS, Feltovich PJ. The clinical reasoning process. Med Educ 1987;21:86-91.

7. de Groot AD. Thought and Choice in Chess. 2nd ed. The Hague: Mouton Publishers, 1978

8. Chase WH, Simon HA. Perception in chess. Cognit Psychol 1973;4:55-81.

9. Eva KW, Norman GR, Neville AJ, Wood TJ, Brooks LR. Expert-novice differences in memory: A reformulation. Teach Learn Med 2002;14:257-263.

10. Ericsson KA, Kintsch W. Long-term working memory. Psychol Rev 1995;102:211-245.

11. Ericsson KA. Deliberate practice and the acquisition and maintenance of expert performance in medicine and related domains. Acad Med 2004;79:S70-S81.

12. Norman GR, Trott AL, Brooks LR, Smith EKM. Cognitive differences in clinical reasoning related to postgraduate training. Teach Learn Med 1994;6:114-120.

13. Eva KW, Neville AJ, Norman GR. Exploring the etiology of content specificity: Factors influencing analogic transfer and problem solving. Acad Med 1998;73:S1-S5.

14. Elstein AS, Shulman LS, Sprafka SA. Medical Problem Solving: An Analysis of Clinical Reasoning. Cambridge, MA: Harvard University Press, 1978

15. Young JQ, van Merrienboer J, Durning S, ten Cate O. Cognitive load theory: Implications for medical education: AMEE Guide No. 86. Med Teach 2014;36:371-384. [http://dx.doi.org/10.3109/0142159X.2014.889290]

16. Simon HA. How big is a chunk? Science 1974;183:482-488.

17. Sweller J, van Merrienboer JJG, Paas FGWC. Cognitive architecture and instructional design. Educ Psychol Rev 1998; 10:251-296.

18. Mayer RE. Multimedia Learning. Cambridge, UK: Cambridge University Press, 2001.

19. Sweller J. Cognitive load theory, learning difficulty, and instructional design. Learn Instr 1994;4:295-312.

20. Mayer RE. Applying the science of learning to medical education. Med Educ 2010;44:543-549. [http://dx.dol org/10.1111/j.1365-2923.2010.03624.x

21. Nielsen J. Usability 101: Introduction to usability. Nielsen Norman Group. 2003. http://www.nngroup.com/ articles/usability-101-introduction-to-usability/ (accessed 31 August 2014).

22. Abran A, Khelifi A, Suryn W, Seffah A. Usability meanings and interpretations in ISO standards. Software Qual J 2003;11:325-338.

23. Sandars I. The importance of usability testing to allow e-learning to reach its potential for medical education. Educ Prim Care 2010;21:6-8.

24. Zaharias P. Usability in the context of e-learning. Int J Technol Human Interact 2009;5:37-59.

Tesource forning re electrolyte and acid-base disorders. Adv Physiol Educ 2011;35:295-306. [http://dx.doi.org/10.1152/advan.00127.2010]
26. Davids MR, Chikte U, Grimmer-Somers K, Halperin ML. Usability testing of a multimedia e-learning resource for electrolyte and acid-base disorders. Brit I Educ Technol 2014;45:367-381.

7. Avouris NM, Dimitracopoulou A, Daskalaki S, Tselios NK. Evaluation of distance-learning environments: Impact of usability on student performance. International Journal of Educational Telecommunications 2001;7:355-378. 28. Kanuka H, Szabo M. Conducting research on visual design and learning: Pitfalls and promises. Can J Lear Technol 1999;27:105-123.

29. Hancock PA, Pepe AA, Murphy LL. Hedonomics: The power of positive and pleasurable ergonomics. Ergon Des 2005;13:8-14.

30. Zaharias P, Poylymenakou A. Developing a usability evaluation method for e-learning applications: Beyond functional usability. Int J Hum-Comput Interact 2009;25:75-98

31. Davids MR, Chikte UME, Halperin ML. An efficient approach to improve the usability of e-learning resources: The role of heuristic evaluation. Adv Physiol Educ 2013;37:242-248. [http://dx.doi.org/10.1152/advan.00043.2013]

32. Davids MR, Chikte UM, Halperin ML. Effect of improving the usability of an e-learning resource: A randomized trial. Adv Physiol Educ 2014;38:155-160. [http://dx.doi.org/10.1152/advan.00119.2013]

33. Nielsen J, Mack RL. Usability Inspection Methods. New York: Wiley, 1994.

34. Dumas JS, Salzman MC. Usability assessment methods. Rev Hum Factors Ergon 2006;2:109-140.

35. Nielsen J. 10 Usability heuristics for user interface design. Nielsen Norman Group. 1995, http://www.nngroup com/articles/ten-usability-heuristics/ (accessed 14 October 2014).

36. Hertzum M, Jacobsen NE. The evaluator effect: A chilling fact about usability evaluation methods. Int J HumComput Interact 2001;13:421-443.

37. Karat C-M, Campbell R, Fiegel T. Comparison of empirical testing and walkthrough methods in user interface evaluation. Proceedings of the SIGCHI Conference on Human Factors in Computing Systems. Monterey, CA ACM, 1992:397-404.

38. Rosenbaum S, Rohn JA, Humburg J. A toolkit for strategic usability: Results from workshops, panels, and surveys. Proceedings of the SIGCHI conference on Human Factors in Computing Systems. The Hague, The Netherlands: ACM 2000:337-344.

39. Hornbæk K. Current practice in measuring usability: Challenges to usability studies and research. Int J HumComput St 2006;64:79-102.

40. Mayer RE. Incorporating motivation into multimedia learning. Learn Instr 2014;29:171-173.

41. Nielsen J. How many test users in a usability study? Jakob Nielsen's Alertbox. 2012. http://www.nngroup.com/ articles/how-many-test-users/ (accessed 1 April 2015)

42. Faulkner L. Beyond the five-user assumption: Benefits of increased sample sizes in usability testing. Behav Res Meth Instrum Comput 2003;35:379-383.

43. Hollender N, Hofmann C, Deneke M, Schmitz B. Integrating cognitive load theory and concepts of humancomputer interaction. Comput Hum Behav 2010;26:1278-1288.

44. Association for Computing Machinery. The ACM Guide to Computing Literature. http://librarians.acm.org acm-guide-computing-literature (accessed 18 August 2015. 